\title{
Role of Thyroid Stimulating Immunoglobulins (TSI) in Early Diagnosis of Patients with Euthyroid Graves' Ophthalmopathy
}

\author{
Mohammad Idris ${ }^{1}$, Muhammad Zubair Umer ${ }^{2}$, Eemaz Nathaniel ${ }^{3}$, Muhammad lqbal $^{4}$, Adnan Zar $^{5}$ \\ ${ }^{1,5}$ Lady Reading Hospital, Medical Teaching Institute (MTI), Peshawar, ${ }^{2}$ Mardan Medical Complex, Mardan, \\ ${ }^{3}$ Rehman Medical College, Peshawar, ${ }^{4}$ PINUM cancer Hospital, Faisalabad
}

\begin{abstract}
Purpose: To determine the role of thyroid stimulating immunoglobulin (TSI) in early diagnosis of patients with euthyroid Graves' ophthalmopathy visiting Oculoplastic service of a tertiary care centre.
\end{abstract}

Study Design: Descriptive cross sectional study.

Place and Duration of Study: Oculoplastic service, outpatient department of ophthalmology, Lady Reading Hospital, Peshawar, Pakistan, from August 2015 to August 2016.

Methods: Patients of both gender, and all ages with any one of the signs and symptoms of Grave's Ophthalmopathy at any stage and of any severity of disease, but normal thyroid functions, were included in the study. Patients were selected by non-probability purposive sampling technique. One-way ANOVA test was done to find out relationship of TSI with age and gender.

Results: Among 90 patients included in the study there were $68.9 \%$ females and $31.1 \%$ males. Maximum number of patients were between 41 and 55 years (37.8\%). Thyroid stimulating immunoglobulin (TSI) test was positive in $84 \%$ patients out of which $55.6 \%$ were females and $28.9 \%$ were males. Age group of $41-55$ years had maximum number of positive TSI $(32.2 \%)$ and $>56$ years age group had $2^{\text {nd }}$ most positive TSI $(26.7 \%)$ with the overall significance of 0.969 . The association of TSI with gender showed higher significance than age with $p$ values of 0.142 and 0.908 respectively.

Conclusion: TSI is positive in majority of female Euthyroid Graves' ophthalmopathy patients in age between 41 and 55 years. Early diagnosis with the help of TSI in euthyroid Graves' disease can prevent ocular complications by timely referral to the endocrinologist.

Key Words: Euthyroid Graves' ophthalmopathy, Thyroid Stimulating Immunoglobulin, Thyroid Function Tests.

How to Cite this Article: Idris M, Umer MZ, Nathaniel E, Iqbal M, Zar A. Role of Thyroid Stimulating Immunoglobulins (TSI) in Early Diagnosis of Patients with Euthyroid Graves' Ophthalmopathy. Pak J Ophthalmol. 2020, 36 (4): 418-422.

Doi: https://doi.org/10.36351/pjo.v36i4.1113

Correspondence: Mohammad Idris

Lady Reading Hospital, Medical Teaching Institute (MTI), Peshawar

Email: idrisdaud80@gmail.com

Received: July 29, 2020

Accepted: September 6, 2020

\section{INTRODUCTION}

Thyroid eye disease is a common reason for many patients visiting Oculoplastic clinics because of its cosmetic and vision related problems. Graves' disease is by far the most common cause of hyperthyroidism, with more than $1 \%$ prevalence in the general population. ${ }^{1}$ Thyroid related disorders are more common in females than males and increase with 
increasing age. ${ }^{2}$ The peak incidence of Graves' disease (GD) occurs among patients of $30-60$ years age, with an increased incidence among African Americans. ${ }^{3}$ The euthyroid Graves' Ophthalmopathy occurs in 5$10 \%$ of patients, which is mostly bilateral. ${ }^{4}$

Thyroid Associated Ophthalmopathy (TAO) is an organ specific, autoimmune process, in which the thyroid gland is over stimulated, causing hyperthyroidism. The extra ocular muscles (EOM) and thyroid gland have same antigenic nature that is recognized by the antibodies. Antibodies bind to the EOM and cause swelling behind the eyeball. The orbital and periorbital tissues are involved leading to swelling which has also been reported to be the result of mucopolysaccharide deposition posterior to the eyes, a symptom indirectly associated with Graves' disease. The "orange peel" skin has been attributed to the infiltration of antibodies beneath the skin, causing an inflammatory response and subsequent fibrous plaques.

Euthyroid Graves' Ophthalmopathy causes the characteristic eye symptoms of Graves' Ophthalmopathy, also known as Thyroid Eye Disease (TED) without thyroid dysfunction. The clinical features of Graves' eye disease involve eyelid retraction, proptosis, conjunctival chemosis, periorbital swelling and in advanced cases may result in loss of sight as a result of optic nerve compression. ${ }^{5}$ There is normal thyroid function tests (free T3, free T4, TSH) and elevated Thyroid Stimulating Immunoglobulin (TSI) levels, in patients with euthyroid Graves' Ophthalmopathy. ${ }^{6}$

Euthyroid Graves' Ophthalmopathy results in an immune response in soft tissues of the orbit especially muscles and these abnormal changes result in typical features of Thyroid Eye Disease. ${ }^{7}$ Therefore, TSI is a good indicator of progress of thyroid disease. ${ }^{8} \mathrm{TAO}$ is a common but still least understood element of Graves' disease, which involves inflammation, congestion and soft tissue remodeling of the orbit and unlike majority of autoimmune disorders, has variable severity and a predictable course. ${ }^{6}$ The TSI measurement is a clinically important indicator of disease severity even in chronic quiescent stage of the disease. $^{9}$

The best management strategy of any disease is to timely treat the cause or underlying mechanism. The timely detection of thyroid stimulating immunoglobulin can be helpful in early diagnosis and management of Graves' eye disease before it worsens clinically and affects vision. The rationale of the study was to improve the scarce data about this disease in Pakistan.

The purpose of the study was to determine the role of thyroid stimulating immunoglobulin (TSI) in early diagnosis of patients with euthyroid Graves' ophthalmopathy visiting Oculoplastic service of a tertiary care centre.

\section{METHODS}

This was a cross sectional descriptive study conducted at the Oculoplastics service, outpatient department of ophthalmology of a tertiary care hospital of Peshawar, Pakistan. The total duration of the study was one year from August, 2015 to August, 2016. Informed consent was taken from all the patients. Patients of both gender, and all ages with any one of the signs and symptoms of Graves' Ophthalmopathy at any stage and of any severity of disease, but normal thyroid functions, were included in the study. All patients having abnormal biochemical profile of thyroid functions (having high or low levels of $\mathrm{T}_{3}, \mathrm{~T}_{4}$, and TSH level) or significant renal diseases, hepatic diseases, pulmonary diseases, patients on immunosuppressive therapy and patients having autoimmune diseases were excluded from the study. Patients who received any medical or surgical treatment in the past for any thyroid disorder were also excluded from the study. The study was conducted after approval from hospital ethical and research committee. All patients presented to the Oculoplastic service, out patient department of ophthalmology, lady Reading Hospital Peshawar, who met the inclusion criteria and who were willing to participate in the study were examined. Graves' Ophthalmopathy was diagnosed on the basis of eye examination by a consultant ophthalmologist (Assistant Professor or above with at least five years of professional experience). An informed written consent was obtained from all patients who were included in this study. All patients had normal biochemical profile of thyroid gland (T3, $\mathrm{T}_{4}$, and TSH). The TSI was calculated by using fully automated chemistry analyzer HPLC (High Performance Liquid Chromatography). The values of TSI was expressed in terms of percentage estimating cAMP accumulated in the presence of test immunoglobulin, in comparison with cAMP accumulated in the presence of normal immunoglobulin. Data was entered and analyzed 
through SPSS version 22. Mean and standard deviations were calculated for numerical data like age. The frequency and percentage were calculated for presence of TSI in the euthyroid Graves' ophthalmopathy. All results were presented in the form of tables and graphs. Stratification was done with regard to the age, gender, duration and severity of disease to see the effects on these variables. One-way ANOVA test was also done for TSI with age groups and gender.

\section{RESULTS}

A total of 90 patients were selected. Females were more than males, $68.9 \%$ and $31.1 \%$ respectively. Four age groups were made in which age group of $41-55$ years had $37.8 \%$ patients and more than 56 years of age group had $32.2 \%$ patients (Table 1). Among the 90 patients, $84 \%$ had their thyroid stimulating immunoglobulin (TSI) test positive. Cross tabulation was performed for TSI with Gender. Among all patients, $84.4 \%$ of the males and females had their TSI positive. Out of these, $55.6 \%$ females and $28.9 \%$ males had positive TSI with a $p$ value of 0.139 (Figure 1). Maximum number of positive TSI were among the age groups $41-55$ years $(32.2 \%)$. Patients with more than 56 years had second high percentage of positive TSI (26.7\%), with the overall significance of 0.969 (Table 1). One-way ANOVA test was also done for TSI with age groups and gender. The association of

Table 1: Distribution of Thyroid Stimulating Immunoglobulin among Age Groups.

\begin{tabular}{ccccc}
\hline $\begin{array}{c}\text { Age in } \\
\text { Years }\end{array}$ & \multicolumn{2}{c}{$\begin{array}{c}\text { Thyroid Stimulating } \\
\text { Immunoglobulin } \\
\text { Yes }\end{array}$} & Notal & p value \\
\hline $11-25$ & $(9) 10.0 \%$ & (2) $2.2 \%$ & $(11) 12.2 \%$ & \\
$26-40$ & $(14) 15.6 \%$ & (2) $2.2 \%$ & (16) $17.8 \%$ & \\
$41-55$ & $(29) 32.2 \%$ & (5) $5.6 \%$ & (34) $37.8 \%$ & 0.969 \\
$>56$ & $(24) 26.7 \%$ & (5) $5.6 \%$ & (29) $32.2 \%$ & \\
Total & (76) $84.4 \%$ & (14) $15.6 \%$ & (90) $100.0 \%$ & \\
\hline
\end{tabular}

Table 2: One - Way ANOVA for Age Groups and Gender with TSI.

\begin{tabular}{llcccc}
\hline & & N & Mean & S. D & Sig. \\
\hline \multirow{2}{*}{ Age in } & Yes & 76 & 2.89 & .988 & \\
Years & No & 14 & 2.93 & 1.07 & .908 \\
& Total & 90 & 2.90 & .995 & \\
& Yes & 76 & 1.66 & .478 & \\
Gender & No & 14 & 1.86 & .363 & .142 \\
& Total & 90 & 1.69 & .466 & \\
\hline
\end{tabular}

TSI with gender showed higher significance than age with $\mathrm{p}$ values of 0.142 and 0.908 respectively (Table 2).

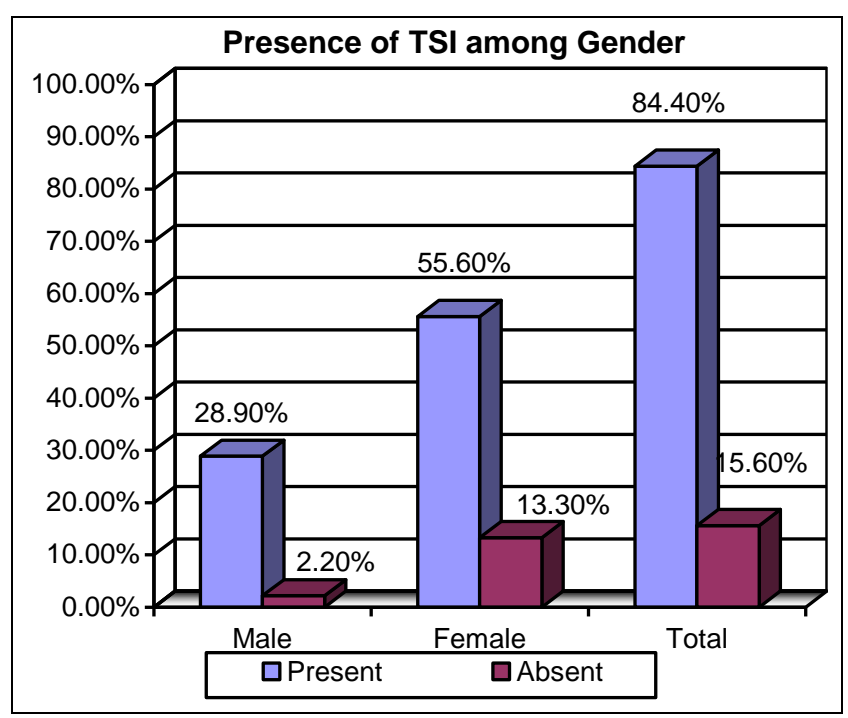

Fig. 1: Gender distribution of thyroid stimulating immunoglobulin $(P$ $=0.139$ ).

\section{DISCUSSION}

Graves' ophthalmopathy (also known as thyroid eye disease, TED, dysthyroid/thyroid-associated orbitopathy (TAO), Graves' orbitopathy) is an autoimmune inflammatory pathology which affects the orbit around the eye, identified by upper eyelid retraction, swelling (edema), redness (erythema), conjunctivitis, and proptosis. ${ }^{10}$ It is thus a cosmetic as well as a sight threatening condition and one of the common reasons for visiting Oculoplastic clinic. Graves' ophthalmopathy is easily diagnosed in a patient with confirmed thyrotoxicosis, positive TSI. ${ }^{11}$

Graves' disease is the most frequent cause of hyperthyroidism in areas with iodine deficiency, with $20-30$ cases annually per 100,000 individuals. ${ }^{1}$ The logical consequence is that patients with Graves' hyperthyroidism, especially elderly, should be given particular attention by general practitioners and thyroid specialists. ${ }^{12}$ It is component of a systemic course with variable expression in the eyes, thyroid and skin, due to binding of auto-antibodies to tissues in these organs. These auto-antibodies target the fibroblasts of eye muscles, which can transform into fat cells (adipocytes). Fat cells and muscles enlarge and become inflamed. Compression of veins fails to drain fluid, resulting in edema. ${ }^{10}$ 
Annual incidence is $16 / 100,000$ in females, $3 / 100,000$ in males. In 3 to $5 \%$ of patients, the disease is severe with intense pain, and sight threatening corneal ulceration or compression of the optic nerve. Tobacco smoking, related with many autoimmune disorders, increases the incidence to 7.7 fold. ${ }^{10}$

Mild disease mostly resolves by using artificial tears and quitting smoking by reducing dryness and discomfort. Treatment of severe cases includes glucocorticoids and occasionally cyclosporine. ${ }^{13}$ Some anti-inflammatory biological mediators, like infliximab, etanercept, and anakinra have been tried, but none of randomized controlled trials have demonstrated effectiveness.

Early diagnosis and timely management can prevent or lessen the complications of this thyroid eye disease. Some patients may present with any one or more features of this thyroid associated ophthalmopathy with thyroid dysfunctions. Some patients although having thyroid functions within normal limits may present with Graves' ophthalmopathy and in some cases, the disease is unilateral. For this presentation patient moves from physician to physician and unnecessary and expensive invasive and non invasive investigations are carried out and all are in vain. The patient thinks that she or he is suffering from some rare disease, which cannot be diagnosed by routine knowledge and investigations. Due to this delay in treatment, some patient undergoes psychological issues and depression, although it is a treatable disease. Majority of signs and symptoms regress and complications are prevented. By evaluating TSI in these Euthyroid patients, which is almost $88.6 \%$ positive in Euthyroid Graves' ophthalmopathy, the early diagnosis and timely treatment can lessen the morbidity. The sensitivity and specificity of TSI is $100 \%$.

Accuna et $\mathrm{al}^{14}$ in their study on 49 patients with Euthyroid Graves' ophthalmopathy, found positive TSI in $65 \%$ of cases which is lower than our study. Possible explanation for this is that we used patients of of all ages and both gender at any stage of the disease. Kraiem et $\mathrm{al}^{15}$ had $72 \%$ positive TSI and again it is lower than in our study. Possible explanation can be better diagnostic tools and newer method of detection.

Emphasis should be given to early diagnosis and timely management of the patients. By estimating TSI, further unnecessary and expensive invasive and noninvasive investigations can be avoided. ${ }^{16}$ In our study,
TSI was present in $88.6 \%$ patients of Euthyroid Graves' ophthalmopathy (sensitivity \& specificity of TSI is $100 \%$ ), and majority of the patients were above 60 years and the duration of disease did not have any effect on severity of disease. Oculoplastic surgeons in particular should be aware of the diagnostic value of TSI to prevent the cosmetic as well as sight threatening complications of this disease by early diagnosis and referral to endocrinologist especially when the disease is in acute stage.

Circulating anti-thyroglobulin (anti-TG) antibodies are also present in Graves' disease but antiTG antibodies can be present in individuals without any other evidence of thyroid dysfunction. ${ }^{17}$ Another important clinical use TSI determinations is their value in predicting the relapse of hyperthyroidism in treated patients. ${ }^{18}$

There are some conflicting reports in the literature regarding TSI levels and Graves' ophthalmopathy. ${ }^{19}$ In a study on children, the authors did not find a relationship between TSI levels and presence of Graves' ophthalmopathy. ${ }^{20}$

Limitation of this study is that it is a single center study and we cannot generalize the results. Further studies are required across Pakistan so that this test can be added in the routine protocol of management of a patient with clinically diagnosed Euthyroid Graves' disease.

\section{CONCLUSION}

TSI is positive in majority patients of Euthyroid Graves' ophthalmopathy, which is a sight-threatening condition in patients visiting Oculoplastic clinics and can be diagnosed early with the help of TSI without any expensive investigations even when other thyroid function tests are normal. The association of TSI with gender showed higher significance than age. Early diagnosis can prevent complications by timely referral to the endocrinologist.

\section{REFERENCES}

1. Smith TJ, Hegedüs L. Graves' Disease. N Engl J Med. 2016; 375 (16): 1552-1565.

2. De Leo S, Lee SY, Braverman LE. Hyperthyroidism. Lancet. 2016; 388 (10047): 906-918.

3. Kahaly GJ, Bartalena L, Hegedüs L, Leenhardt L, Poppe K, Pearce SH. 2018 European Thyroid Association Guideline for the Management of Graves' Hyperthyroidism. Eur Thyroid J. 2018; 7 (4): 167-186. 
4. Arslan E, Yava ÅÿoÄŸlu I, Cilda ÄŸ BM, Kocatürk T. Unilateral optic neuropathy as the initial manifestation of euthyroid Graves' disease. Intern Med. 2009; 48 (22): 1993-1994.

5. Weiler DL. Thyroid eye disease: a review. Clin Exp Optom. 2017; 100 (1): 20-25.

6. Suzuki N, Noh JY, Kameda T, Yoshihara A, Ohye H, Suzuki M, et al. Clinical course of thyroid function and thyroid associated-ophthalmopathy in patients with euthyroid Graves' disease. Clin Ophthalmol. 2018; 12: 739-746.

7. Khanzada TW, Memon W, Kumar B, Samad A. Thyroid Scintigraphy: an overused investigation. Gomal J Med Sci. Jan - Jun. 2009; 7 (1): 39-41.

8. Tay WL, Chng CL, Tien CS, Loke KS, Lam WW, Fook-Chong SM, et al. High Thyroid Stimulating Receptor Antibody Titre and Large Goitre Size at FirstTime Radioactive Iodine Treatment are Associated with Treatment Failure in Graves' Disease. Ann Acad Med Singapore, 2019; 48 (6): 181-187.

9. Woo YJ, Jang SY, Lim TH, Yoon JS. Clinical Association of Thyroid Stimulating Hormone Receptor Antibody Levels with Disease Severity in the Chronic Inactive Stage of Graves' Orbitopathy. Korean J Ophthalmol. 2015; 29 (4): 213-219.

10. Grebe SKG. Thyroid disease. In The Genetic Basis of Common Diseases. Second edition. Edited by RA King, JI Rotter, AG Motulsky. New York, Oxford University Press, 2002: pp 397-430.

11. Subekti I, Pramono LA. Current Diagnosis and Management of Graves' Disease. Acta Med Indones. 2018; 50 (2): 177-182.

12. Bartalena L, Chiovato L, Marcocci C, Vitti P, Piantanida E, Tanda ML. Management of Graves' hyperthyroidism and orbitopathy in time of COVID-19 pandemic [published online ahead of print, 2020 May 21]. J Endocrinol Invest. 2020: 1-3.

13. Cakir M. Euthyroid Graves' ophthalmopathy with negative autoantibodies. J Natl Med Assoc. 2005; 97 (11): 1547-1549.

14. Acuna OM, Athannassaki I, Paysse EA. Association between thyroid-stimulating immunoglobulin levels and ocular findings in pediatric patients with Graves disease. Trans Am Ophthalmol Soc. 2007; 105: 146-151.

15. Kraiem Z, Glaser B, Pauker J, Sadeh O, Sheinfeld M. Bioassay of thyroid stimulating immunoglogulin in cryopreserved human thyroid cells: optimization and clinical evaluation. J Clin Chem. 1988; 34 (2): 244-249.
16. Kiran Z, Rashid O, Islam N. Typical graves' ophthalmopathy in primary hypothyroidism. J Pak Med Assoc. 2017; 67 (7): 1104-1106.

17. Hollowell JG, Staehling NW, Flanders WD, Hannon WH, Gunter EW, Spencer CA, et al. Serum TSH, T(4), and thyroid antibodies in the United States population (1988 to 1994): National Health and Nutrition Examination Survey (NHANES III). J Clin Endocrinol Metab. 2002; 87 (2): 489-499.

18. Pinchera A, Fenzi GF, Macchia E, Bartalena L, Mariotti S, Monzani F. Thyroid-stimulating immunoglobulins. Horm Res. 1982; 16 (5): 317-328. Doi: 10.1159/000179520.

19. Dragan LR, Seiff SR, Lee DC. Longitudinal correlation of thyroid-stimulating immunoglobulin with clinical activity of disease in thyroid-associated orbitopathy. Ophthal Plast Reconstr Surg. 2006; 22: 13-19.

20. Shibayama K, Ohyama Y, Yokota Y, Ohtsu S, Takubo N, Matsuura N. Assays for thyroidstimulating antibodies and thyrotropin-binding inhibitory immunoglobulins in children with Graves' disease. Endocr J. 2005; 52 (5): 505-510.

\section{Authors' Designation and Contribution}

Mohammad Idris; Assistant Professor: Concepts, Design, Literature research, Data acquisition, Data analysis, Manuscript preparation, Manuscript editing, Manuscript review

Muhammad Zubair Umer; Assistant Professor Pathology: Concepts, Design, Literature research, Data acquisition, Manuscript preparation, Manuscript editing

Eemaz Nathaniel; Editor JRMI: Literature research, Data acquisition, Statistical analysis, Manuscript preparation, Manuscript editing

Muhammad Iqbal; Consultant Ophthalmologist: Literature research, Data acquisition

Adnan Zar; SPR: Data analysis, Manuscript review. 